\title{
Classification of High-Grade Glioma into Tumor and Nontumor Components Using Support Vector Machine
}

\author{
(DD.T. Blumenthal, (D) M. Artzi, (D) G. Liberman, F. Bokstein, DO. Aizenstein, and DD. Ben Bashat
}

\begin{abstract}
BACKGROUND AND PURPOSE: Current imaging assessment of high-grade brain tumors relies on the Response Assessment in NeuroOncology criteria, which measure gross volume of enhancing and nonenhancing lesions from conventional MRI sequences. These assessments may fail to reliably distinguish tumor and nontumor. This study aimed to classify enhancing and nonenhancing lesion areas into tumor-versus-nontumor components.
\end{abstract}

MATERIALS AND METHODS: A total of 140 MRI scans obtained from 32 patients with high-grade gliomas and 6 patients with brain metastases were included. Classification of lesion areas was performed using a support vector machine classifier trained on 4 components: enhancing and nonenhancing, tumor and nontumor, based on T1-weighted, FLAIR, and dynamic-contrast-enhancing MRI parameters. Classification results were evaluated by 2-fold cross-validation analysis of the training set and MR spectroscopy. Longitudinal changes of the component volumes were compared with Response Assessment in Neuro-Oncology criteria.

RESULTS: Normalized T1-weighted values, FLAIR, plasma volume, volume transfer constant, and bolus-arrival-time parameters differentiated components. High sensitivity and specificity (100\%) were obtained within the enhancing and nonenhancing areas. Longitudinal changes in component volumes correlated with the Response Assessment in Neuro-Oncology criteria in 27 patients; 5 patients (16\%) demonstrated an increase in tumor component volumes indicating tumor progression. These changes preceded Response Assessment in Neuro-Oncology assessments by several months. Seven patients treated with bevacizumab showed a shift to an infiltrative pattern of progression.

CONCLUSIONS: This study proposes an automatic classification method: segmented Response Assessment in Neuro-Oncology criteria based on advanced imaging that reliably differentiates tumor and nontumor components in high-grade gliomas. The segmented Response Assessment in Neuro-Oncology criteria may improve therapy-response assessment and provide earlier indication of progression.

ABBREVIATIONS: BAT = bolus arrival time; $\mathrm{DCE}=$ dynamic contrast-enhanced; $\mathrm{GB}=$ glioblastoma; $\mathrm{HGG}=$ high-grade gliomas; $k_{\text {ep }}=$ interstitium-to-plasma rate constant; $K^{\text {trans }}=$ volume transfer constant; NAWM = normal-appearing white matter; nFLAIR = normalized FLAIR images; nTIWI+Gd = normalized Tl-weighted images post-contrast agent; $v_{e}=$ interstitial volume; $v_{p}=$ plasma volume; RANO = Response Assessment in Neuro-Oncology; SPGR = spoiled gradient-recalled; sRANO = segmented RANO; SVM = support vector machine

$\mathrm{H}$ gh-grade gliomas (HGG), specifically glioblastoma (GB), remain the most common and aggressive brain tumors in adults. Despite recent advances in treatment, long-term survival remains low. ${ }^{1,2}$ Treatment of newly diagnosed and recurrent

Received October 6, 2016; accepted after revision January 3, 2017

From the Neuro-Oncology Service (D.T.B., F.B.) and Functional Brain Center (M.A., O.A., D.B.B.), Tel Aviv Sourasky Medical Center, Tel Aviv, Israel; Sackler Faculty of Medicine (D.T.B., M.A., F.B., D.B.B.) and Sagol School of Neuroscience (D.B.B.), Tel Aviv University, Tel Aviv, Israel; and Department of Chemical Physics (G.L.), Weizmann Institute, Tel Aviv, Israel.

D.T. Blumenthal and M. Artzi contributed equally to this work.

Paper previously presented in part at: Annual Meeting and Exhibition of the International Society for Magnetic Resonance in Medicine, May 7-13, 2016; Singapore.

Please address correspondence to Dafna Ben Bashat, PhD, Functional Brain Center, Tel Aviv Sourasky Medical Center, 6 Weizman St, Tel-Aviv 64239 Israel; e-mail: dafnab@tlvmc.gov.il
HGG consists of a combination of surgery, radiation therapy, and chemotherapy and, more recently, biologic and immunotherapies, some of which are under clinical investigation. Unique imaging phenomena characterize therapeutic responses to these therapies, challenging conventional radiologic interpretation.

MRI is the method of choice for the initial diagnosis, follow-up, and therapy response assessment of brain tumors. HGG are typically characterized by contrast-enhanced areas on T1-weighted imaging and hyperintensity on FLAIR and T2WI. The current standard for the radiologist's assessment in

\footnotetext{
- Indicates open access to non-subscribers at www.ajnr.org

三 Indicates article with supplemental on-line appendix.

Indicates article with supplemental on-line photo.

http://dx.doi.org/10.3174/ajnr.A5127
} 
patients with HGG relies on the Response Assessment in Neuro-Oncology (RANO) criteria, ${ }^{3}$ which expand upon the earlier Macdonald ${ }^{4}$ criteria, to incorporate the nonenhancing component of the tumor, as this component may indicate infiltrative or diffuse tumor growth. However, the current RANO criteria, which rely on conventional imaging, may fail to distinguish different tissue components that have similar imaging patterns (ie, contrast-enhanced T1WI) yet different prognostic implications (tumor-versus-treatment effects), the identification of which is crucial for appropriate clinical management. ${ }^{3,5}$

Glioblastoma shows high heterogeneity within the lesion area, including areas of active tumor, necrosis, infiltrative tumor cells, and vasogenic edema. Enhancing lesion area can be measured on conventional T1WI; however, it may represent a variety of both tumor and nontumor processes, including tumor progression, pseudoprogression, postsurgical changes, and radiation effects. ${ }^{3}$ Differentiation of the nonenhancing area between infiltrative tumor and nontumor area (edema and/or gliosis) is challenging, as both are characterized by hyperintense FLAIR/T2WI signal. Hyperintense FLAIR/T2WI signal can be expected to represent pure vasogenic edema in metastatic tumors (characteristically with well-defined borders between malignant cells and normal brain), ${ }^{6}$ while in HGG, the hyperintensity may reflect areas of infiltrating tumor cells and/or vasogenic edema.

Glioblastoma is characterized by a highly complex neovascularization process. The increased vascularity of GB allows analysis and characterization of the tumor using advanced vascular MRI methods, including perfusion and permeability. Dynamic contrast-enhanced (DCE) imaging has been widely used for characterization of tumor biology and therapy-response assessment. DCE relies on dynamic T1WI sequences during bolus injection of contrast agent. Hemodynamic parameters, including plasma volume $\left(v_{\mathrm{p}}\right)$ and tissue transfer constants (specifically, volume transfer constant $\left[K^{\text {trans }}\right]$ and interstitium-to-plasma rate constant $\left.\left[k_{\mathrm{ep}}\right]\right)$, can be extracted from DCE data.

Several studies have suggested the utility of DCE parameters as important imaging markers for brain tumor diagnosis, grading, and therapy-response monitoring ${ }^{7-9}$; to differentiate between active and nonactive tumor components ${ }^{10-14}$ or infiltrative and noninfiltrative tumor ${ }^{10,15}$; and for early-stage assessment of antiangiogenic therapy response in HGG. ${ }^{16,17}$ However, none of these studies have aimed to classify the entire lesion area, both enhancing and nonenhancing, into tumor and nontumor areas at the voxel level.

Various techniques have been proposed for segmentation and classification of brain lesions. ${ }^{18}$ Support vector machine (SVM) is a supervised binary classifier, originally aimed at classifying data into 2 classes. Based on a training dataset, the algorithm finds the hyperplane that maximally separates between points. New data are then mapped onto the same space and assigned to a particular class. SVM has been widely used as a classifier of medical images and has proved advantageous over other algorithms of its kind. ${ }^{18,19}$

The aim of this study was to classify lesion areas in patients with HGG using SVM into 4 distinct components: 1) enhancing tumor, 2) enhancing nontumor, 3) nonenhancing tumor, and 4)
Table 1: Patient characteristics

\begin{tabular}{lcccc}
\hline \multicolumn{1}{c}{ Diagnosis } & $\begin{array}{c}\text { No. of } \\
\text { Patients }\end{array}$ & $\begin{array}{c}\text { No. of } \\
\text { Scans }\end{array}$ & Age (yr) & F/M \\
\hline Glioblastoma & 26 & 95 & $52 \pm 14$ & $14: 12$ \\
Anaplastic astrocytoma & 5 & 31 & $45 \pm 12$ & $5: 0$ \\
Anaplastic oligodendroglioma & 1 & 3 & 53 & $0: 1$ \\
Brain metastasis & 6 & 11 & $64 \pm 13$ & $1: 5$ \\
Total & 38 & 140 & & \\
\hline
\end{tabular}

nonenhancing nontumor. Segmentation of lesion areas into tumor and nontumor components refines the RANO criteria and may improve therapy response MRI assessment.

\section{MATERIALS AND METHODS Patients}

A total of 140 MRI scans were obtained from 38 patients, who were scanned every 2-3 months. Ninety-five scans were from 26 patients with GB; 31 scans (5 patients) were of anaplastic astrocytomas; 3 scans ( 1 patient) were of anaplastic oligodendroglias; and 11 scans ( 6 patients) were of brain metastases ( 4 breast and 2 lung cancers) (Table 1). Inclusion criteria required a normal glomerular filtration rate and no contraindication to MRI. All patients with HGG who were scanned longitudinally $(n=26)$ had recurrent HGG and had been treated initially with standard chemoradiation. ${ }^{1}$ Additional therapies are detailed in the On-line Appendix. Nine patients received bevacizumab at recurrence and had pre- and post bevacizumab MRIs.

The study was approved by the Tel Aviv Sourasky Medical Center review board, and written informed consent was obtained from all patients.

\section{MR Imaging Protocol}

Scans were performed on 3T MRI scanners: 91 scans were performed on a Signa Excite scanner (GE Healthcare) using an 8-channel head coil, and 49 scans were performed on a Magnetom Prisma scanner (Siemens) using a 20-channel head coil. The protocol included conventional imaging: spoiled gradient-recalled (SPGR)/FLASH T1WI performed before and after contrast agent injection and FLAIR. DCE data were acquired using multiphase 3D T1WI SPGR/FLASH before, during, and after contrast agent injection (FOV $250 \mathrm{~mm}$; matrix 256X256/256X184, section thickness of $5 \mathrm{~mm}, \mathrm{TR} / \mathrm{TE}=5 / 2.2 \mathrm{~ms}$, and flip angle $=20^{\circ}$. For the T1 maps, variable flip angle SPGR/FLASH data were acquired with nominal flip angles of $5^{\circ}, 10^{\circ}, 15^{\circ}, 20^{\circ}$, and $30^{\circ}$. Dynamic data were acquired with a temporal resolution of 6 seconds and scan duration of 6 minutes. A power injector was used to infuse a single dose $(0.2 \mathrm{~mL} / \mathrm{kg})$ of contrast agent (gadoterate meglumine, 0.5 $\mathrm{mol} / \mathrm{L}$, Dotarem; Guerbet, Aulnay-sous-Bois, France) followed by $20 \mathrm{~mL}$ saline flush, both at a constant rate of $5 \mathrm{~mL} / \mathrm{s}$ second. Fourteen-to-twenty sections were centered on the tumor area as identified in the conventional images, providing brain coverage of $70-100 \mathrm{~mm}$. MR spectroscopy data were acquired with a singlevoxel point-resolved sequence by using a cubic volume of $\sim 8 \mathrm{~mL}$ located at the lesion area and normal-appearing white matter (NAWM) in the contralateral hemisphere $(\mathrm{TR} / \mathrm{TE}=1500 / 35$ $\mathrm{ms})$.

AJNR Am J Neuroradiol 38:908-14 May 2017 www.ajnr.org 909 
Table 2: Mean and SD of the extracted MR imaging parameters between lesion components

\begin{tabular}{|c|c|c|c|c|c|}
\hline & \multirow[b]{2}{*}{ NAWM } & \multicolumn{2}{|c|}{ Enhancing } & \multicolumn{2}{|c|}{ Nonenhancing } \\
\hline & & Tumor & Nontumor & Tumor & Nontumor \\
\hline $\mathrm{nTIWI}+\mathrm{Gd}$ & $0.010 \pm 0.000$ & $0.019 \pm 0.005^{a}$ & $0.013 \pm 0.001^{\mathrm{a}}$ & $0.009 \pm 0.001^{b}$ & $0.007 \pm 0.001^{b}$ \\
\hline nFLAIR & $0.010 \pm 0.000$ & $0.017 \pm 0.005$ & $0.015 \pm 0.004$ & $0.014 \pm 0.002^{\mathrm{b}}$ & $0.018 \pm 0.003^{b}$ \\
\hline$v_{p}$ & $0.010 \pm 0.001$ & $0.054 \pm 0.030^{a}$ & $0.007 \pm 0.003^{\mathrm{a}}$ & $0.021 \pm 0.005^{b}$ & $0.004 \pm 0.001^{\mathrm{b}}$ \\
\hline$K^{\text {trans }}$ & $0.001 \pm 0.001$ & $0.064 \pm 0.037^{a}$ & $0.006 \pm 0.004^{\mathrm{a}}$ & $0.009 \pm 0.005^{\mathrm{b}}$ & $0.000 \pm 0.000^{b}$ \\
\hline$k_{\text {ep }}$ & - & $0.016 \pm 0.011^{\mathrm{a}}$ & $0.002 \pm 0.003^{\mathrm{a}}$ & - & $\begin{array}{c}0.000- \\
-\end{array}$ \\
\hline$v_{e}$ & - & $0.012 \pm 0.009^{a}$ & $0.001 \pm 0.001^{\mathrm{a}}$ & - & - \\
\hline $\begin{array}{l}e \\
\text { BAT }\end{array}$ & $1.00 \pm 0.20$ & $1.30 \pm 0.20$ & $1.20 \pm 0.30$ & $1.20 \pm 0.20^{b}$ & $0.90 \pm 0.40^{\mathrm{b}}$ \\
\hline $\mathrm{NAA}+\mathrm{NAAG} / \mathrm{Cr}+\mathrm{PCh}$ & $1.52 \pm 0.25$ & $1.32 \pm 0.70$ & - & 1.35 & $1.04 \pm 0.22$ \\
\hline $\mathrm{GPC}+\mathrm{PCh} / \mathrm{Cr}+\mathrm{PCh}$ & $0.30 \pm 0.06$ & $0.70 \pm 0.25$ & - & 0.46 & $0.30 \pm 0.05$ \\
\hline
\end{tabular}

Note:-NAAG indicates N-acetyl aspartylglutamate; GPC, glycerolphosphocholine; PCho, phosphocholine.

a Significant difference $(P<.05)$ between the 2 enhancing components.

b Significant difference $(P<.05)$ between the 2 nonenhancing components.

\section{Lesion Classification}

Lesion classification involved several steps as detailed in the Online Appendix and shown in On-line Fig 1. Following preprocessing, the enhancing and nonenhancing lesion areas, designated as targets for classification, were automatically segmented from the T1WI and FLAIR (respectively) images by using an adaptive threshold as previously described. ${ }^{20}$ Manual correction was performed when necessary. Normalized images of FLAIR (nFLAIR) and postcontrast T1WI (nT1WI+Gd) were calculated relative to NAWM. The DCE pharmacokinetic parameters, $v_{\mathrm{p}}, K^{\text {trans }}$, interstitial volume $\left(v_{\mathrm{e}}\right), k_{\mathrm{ep}}$, and bolus arrival time (BAT), were estimated by using DCE-Up-Sampled-Temporal-Resolution (DUSTER). ${ }^{21}$

Classification of the lesion area into 4 components was performed automatically by using the SVM. For the training data, volumes of interest of 5-10 voxels each $(30-60 \mathrm{cc})$ were manually defined with the FMRIB Software Library (FSL; http://www. fmrib.ox.ac.uk/fsl) in areas with known pathology for the 4 tissue types: 1) enhancing tumor, defined retrospectively in patients with progressive HGG based on the RANO assessment on follow-up scans; 2) enhancing nontumor, defined retrospectively in patients with nonprogressive $\mathrm{HGG}$, with treatment-related changes based on stable longitudinal ( $>6$-month) RANO assessments; 3 ) nonenhancing tumor (infiltrative), defined as a peritumoral area ( $<2 \mathrm{~cm}$ from the enhanced tumor margins) in a nonenhancing FLAIR hyperintense region in patients with HGG with progressive disease; and 4) nonenhancing, nontumor (edema), defined in patients with brain metastasis, in whom the hyperintense FLAIR signal represented pure vasogenic edema, without tumor cell infiltration. ${ }^{6}$

Seven MRI parameters were measured within and compared between tissue types: nT1WI+Gd, nFLAIR, $v_{\mathrm{p}}, K^{\text {trans }}, v_{\mathrm{e}}, k_{\mathrm{ep}}$, and BAT. SVM classifier with a linear kernel was trained only on the MRI parameters that were found to be significantly different $(P<$ .05 ) among the tissue types. Voxelwise classification using SVM was performed separately for the enhancing and nonenhancing lesion areas in all patients.

\section{Evaluation}

Two-Fold Cross-Validation Analysis. To test the sensitivity and specificity of the method with validating confidence intervals, we performed 300 iterations of the training and testing data based on different random selections of the even and odd partitions. The group was divided into 2 subgroups (randomized, even-versus- odd) with 1 subgroup as training data, the other as the test data, and vice versa.

MR Spectroscopy. Classification results were validated based on MR spectroscopy results, performed off-line using LCModel (http://www.lcmodel.com/). ${ }^{22}$ Metabolite ratios of glycerophosphocholine + phosphocholine (GPC+PCho) and of $\mathrm{N}$-acetyl aspartate $+\mathrm{N}$-acetyl aspartylglutamate $(\mathrm{NAA}+\mathrm{NAAG})$ relative to creatine + phosphocreatine $(\mathrm{Cr}+\mathrm{PCr})$ were calculated. While many MR spectra were acquired for clinical purposes, MR spectroscopy data were used in this study when voxel location included only 1 tissue component or were defined within the NAWM.

Longitudinal Assessment. Longitudinal assessment of the classification results was performed in patients with primary brain tumors (32 patients). Longitudinal changes in the volume of each component were evaluated relative to RANO criteria assessed by a senior neuroradiologist defining 4 categories: complete response, partial response, stable disease, or progression. ${ }^{3}$

\section{Statistical Analysis}

To identify differences between tumor and nontumor components for the various MRI parameters (separately for the enhancing and nonenhancing area), we used the Wilcoxon test (because parameters were not normally distributed).

\section{RESULTS \\ Classification Results}

Training Data. We defined VOIs for the 4 tissue types: the enhancing tumor component in 28 scans from 5 patients with progressive HGG (4 GB and 1 anaplastic astrocytoma); the enhancing nontumor component in 10 scans from 3 patients with high-grade tumors ( $2 \mathrm{~GB}$ and 1 oligodendroglioma); the nonenhancing tumor component in 24 scans from 5 patients with GB; and the nonenhancing, nontumor component in 11 scans from 6 patients with brain metastasis.

Mean values of the 7 MRI parameters, nT1WI+Gd, nFLAIR, $v_{\mathrm{p}}, K^{\text {trans }}, v_{\mathrm{e}}, k_{\mathrm{ep}}$, and BAT, measured in the 4 components (tissue types) defined for the training data and NAWM, are provided in Table 2. For the enhancing components, significant differences were detected for the $\mathrm{nT} 1 \mathrm{WI}+\mathrm{Gd}, v_{\mathrm{p}}, k^{\text {trans }}, k_{\mathrm{ep}}$, and $v_{\mathrm{e}}$ parameters, demonstrating higher vascularity $\left(v_{\mathrm{p}}\right)$ and impaired permeability $\left(\mathrm{nT} 1 \mathrm{WI}+\mathrm{Gd}, K^{\text {trans }}\right)$ in the tumor component relative to 
the nontumor component. For the nonenhancing components, significant differences between components were detected for all parameters, demonstrating higher vascularity $\left(v_{\mathrm{p}}\right)$ and slightly impaired permeability (nT1WI $\left.+\mathrm{Gd}, K^{\text {trans }}\right)$ in the tumor component relative to the nontumor component. The $v_{\mathrm{e}}$ and $k_{\mathrm{ep}}$ parameters showed significant group differences yet a substantially high SD $(>150 \%)$ and were therefore not used in the training set.

\section{Classification of Lesion Area}

The SVM classifier was trained on the nT1WI+Gd, $v_{\mathrm{p}}$, and $K^{\text {trans }}$ parameters for enhancing components and on the nT1WI+Gd, nFLAIR, $v_{\mathrm{p}}, K^{\text {trans }}$, and BAT parameters for nonenhancing components. Voxelwise classification was performed on all scans of all patients $(n=140)$, separately for enhancing and nonenhancing lesion areas. Figure 1 shows classification results obtained in 3 patients. In the first patient (Fig 1, case 1) with breast cancer metastasis, the non-
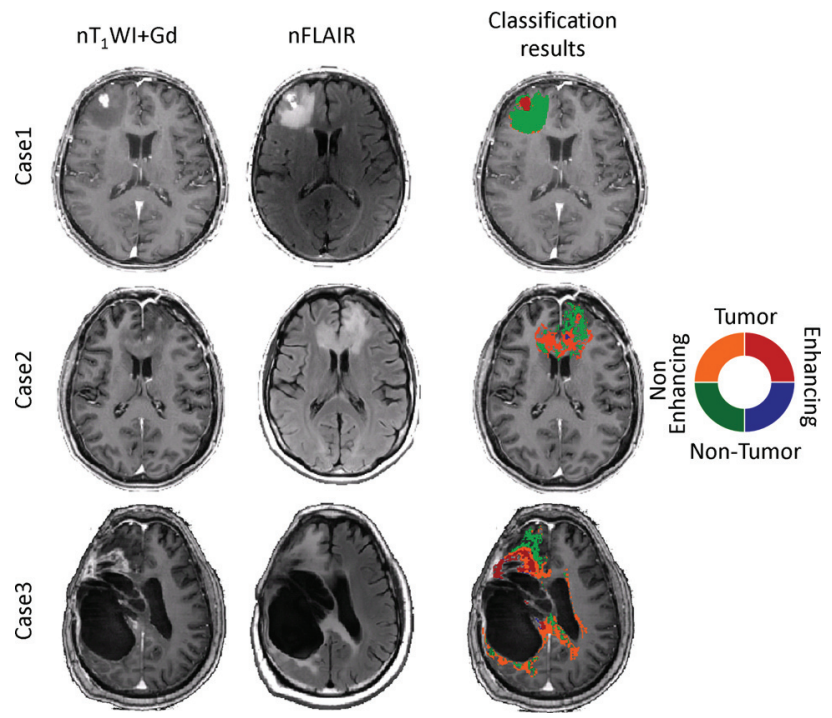

FIG 1. Classification results obtained in a patient with active breast cancer metastasis (case 1) and in 2 patients with glioblastoma (cases 2 and 3).
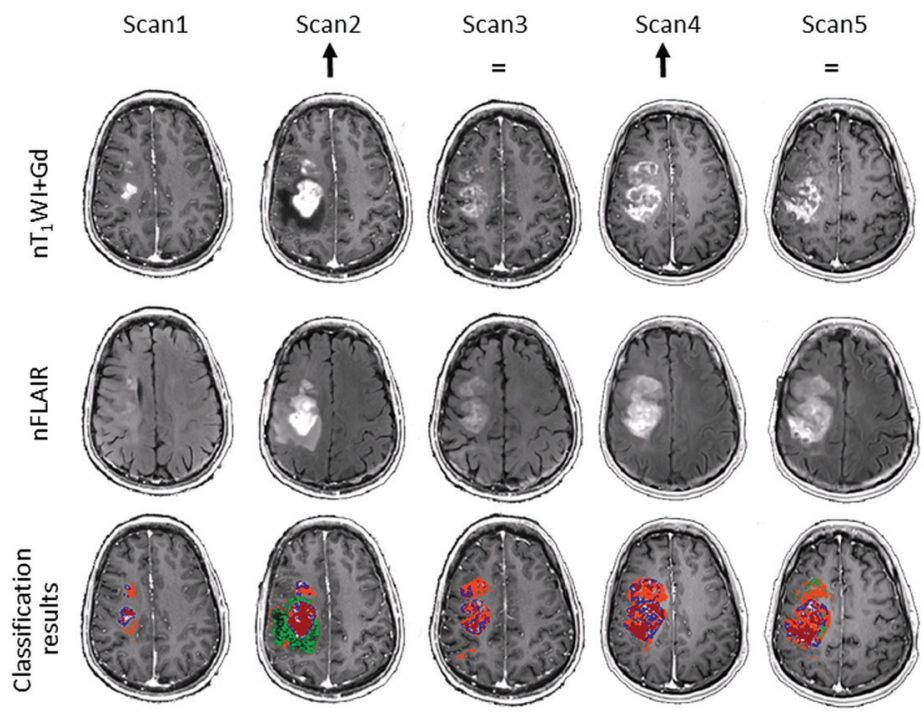

FIG 2. Longitudinal classification and radiologic results obtained in a 54-year-old patient with anaplastic astrocytoma (patient 18) scanned longitudinally every 2 months.
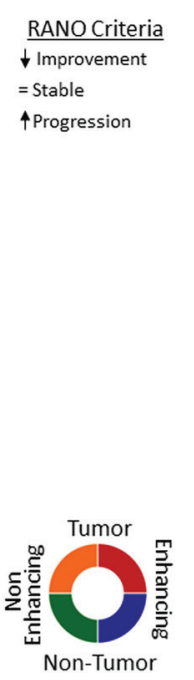

enhancing lesion area was classified as nonenhancing, nontumor (ie, edema) and the enhancing lesion area was classified as enhancing tumor. The second patient (Fig 1, case 2) with GB was an example in whom most of the nonenhancing lesion was classified as tumor component. This patient was diagnosed with progressive disease on a subsequent follow-up scan (2 months later). In the third patient (Fig 1, case 3), also with GB, the enhancing lesion and most of the nonenhancing area were classified as tumor component, consistent with a diagnosis of progressive disease as diagnosed on subsequent follow-up.

\section{Longitudinal Assessment}

Longitudinal assessment of the volume of each component and the correlating radiologic assessment based on RANO, for 26 patients with high-grade lesions scanned longitudinally $>3$ times (a total of 118 scans) are shown in On-line Fig 2. Consistent results were obtained between changes in the volume of the lesion components and the radiologist's assessment in 27 patients (of 32, $84 \%$ ). However, in 5 (16\%; patients $1,17,18,20$, and 26 ), increased volume of the nonenhancing tumor component indicating tumor progression preceded the radiologic diagnosis based on RANO criteria by several months.

Figure 2 shows longitudinal data obtained from a 54-year-old patient with anaplastic astrocytoma (patient 18). While the conventional imaging showed a pattern of stability at scans 3 and 5, with reduction/no substantial changes in the enhancing and nonenhancing lesion areas, classification results revealed a different clinical scenario-progressive tumor growth. A continual increase in the nonenhancing tumor component indicated a pattern of infiltrative disease progression through scans $2-5$ (particularly from scan 2 to 3 ). This patient was diagnosed with progressive disease at scan 4 and died 71 days following scan 5 .

In 7 of 9 patients who received bevacizumab, substantial reductions (mean, 56\%) were detected mainly in the volume of the nonenhancing, nontumor component (interpreted as edema). Three patients of 7 (patients 12,13, and 23), though showing a major reduction in the volume of the nonenhancing, nontumor component, had an increase in the nonenhancing tumor component, suggesting a shift to an infiltrative pattern of tumor progression during bevacizumab treatment.

\section{Evaluation}

Sensitivity and Specificity of the Training Data. Analysis demonstrated 100\% sensitivity $(85 \% / 100 \%$ for the 5 th/ 90 th percentiles) and $100 \%$ specificity (100\%/ $100 \%$ ) for the identification of enhancing tumor and enhancing nontumor components, and 100\% sensitivity (100\%/ $100 \%)$ and $100 \%$ specificity (100\%/ $100 \%$ ) for identification of the nonenhancing (infiltrative) tumor and nonenhancing nontumor components.

MR Spectroscopy Results. Forty-one spectra were obtained with good quality, 
with voxel location consisting of 1 of the 4 components or within NAWM: 14 from NAWM; 6 from nonenhancing, nontumor component; 1 from nonenhancing tumor; and 19 from enhancing tumor components. MR spectroscopy results are provided in Table 2. Spectra from the enhancing, nontumor components showed primarily lipid peaks, without a detectable metabolite ratio. MR spectroscopy supported the classification results, demonstrating a $>1.5$-fold increase in glycerolphosphocholine + phosphocholine/creatine + phosphocholine $(\mathrm{GPC}+\mathrm{PCh} / \mathrm{Cr}+\mathrm{PCr})$ ratio in the tumor components relative to the nontumor component and NAWM.

\section{DISCUSSION}

In this study, longitudinal classification of lesion area into tumor and nontumor components was performed in patients with HGG based on multi-MRI parameters extracted from conventional imaging and DCE MRI. RANO criteria improved the earlier Macdonald ${ }^{4}$ criteria by incorporating the nonenhancing component of the lesion. Our proposed method, referred to as segmented RANO (sRANO), provides a logical next step in the evolution of MR clinical imaging, to better assess tumor growth and therapy response. Both enhancing and nonenhancing lesion areas may include tumor and nontumor components. The proposed sRANO classifies the lesion areas and defines each component separately. In $16 \%$ of our cases followed longitudinally, we were able to identify tumor progression several months in advance of RANO criteria.

Conventional MRI lacks the precision to reliably differentiate tumor and nontumor areas with similar imaging patterns. However, the implications of such differentiation regarding treatment are substantial. Within the enhancing lesion area, recognition of dynamic, active tumor typically requires a change in therapy. Likewise, the identification of radiation necrosis or treatmentrelated changes can prevent unnecessary interventions. While salvage re-irradiation of brain tumors can typically be performed with a minimum of resultant treatment necrosis, ${ }^{23}$ re-irradiation to an area of preexisting treatment-related necrosis could exacerbate existing tissue damage and lead to potentially life-threatening neurologic toxicity.

Classification between tumor and nontumor areas within the nonenhancing lesion may provide new guidelines for radiation. Standards of treatment-planning techniques differ geographically. ${ }^{24}$ Accurate delineation of tumor area may either limit the size of the radiation field and decrease radiation exposure to nontumor brain tissue or define more accurately the optimal radiation field to include the entire tumor area and enable more efficient treatment.

In addition, as the use of immune-mediating therapies in glioma increases, the complexity of interpreting inflammatory responses to treatment ("pseudoprogression") in enhancing and nonenhancing lesion areas will also increase. Misinterpretation of the response to a potentially helpful therapy could lead to premature cessation of a useful therapy and compromise patient outcome. $^{25}$

Within the enhancing lesion area, all measured vascular parameters (except the BAT) clearly differentiated tumor and nontumor components. While both components demonstrated increased tissue permeability, the tumor component was characterized by much higher permeability and $v_{\mathrm{p}}$ compared with the nontumor component and relative to NAWM. GB is characterized by a complex neovascularization process that results in formation of new, abnormal blood vessels and an impaired blood-brain barrier, and thus manifests as a hyperperfused area with increased blood flow, volume, and permeability, as was seen in our cohort study. ${ }^{26,27}$ The nontumor component demonstrated reduced $v_{\mathrm{p}}$ compared with NAWM, consistent with data that have shown areas of treatment-related changes characterized by impaired blood brain barrier yet with reduced blood flow and volume, due to treatment-induced vascular endothelial damage and coagulative necrosis. ${ }^{5,28}$

Within the nonenhancing lesion area, despite a similar appearance between tumor and nontumor components on conventional imaging (FLAIR and T2WI), significant differences were found in all measured MRI parameters. The nonenhancing, nontumor component was characterized by reduced $v_{\mathrm{p}}$, in comparison with the NAWM and the nonenhancing tumor component. This pattern is consistent with previous reports of tissues proximal to brain tumors in patients ${ }^{10,29,30}$ and animal models ${ }^{31}$ and can be explained by compression of regional capillaries caused by vasogenic edema. ${ }^{32}$ The tumor component in our cohort was characterized by increased tissue permeability and increased perfusion as demonstrated by $K^{\text {trans }}$ and $v_{\mathrm{p}}$ parameters, consistent with findings in previous studies. ${ }^{10,29,33}$

In this work, classification of lesion area was performed with a linear SVM classifier, a simple form of SVM, highly suitable for a limited training set size, for which the separating hyperplane is simply a plane in the feature space and the relative contribution of the features can be easily obtained. Various machine learningand computational intelligence-based methods have been proposed for segmentation of brain tumors. ${ }^{34}$ In this study, SVM was found to be a robust, rapid method, tailored to the clinical data, easily implemented, and most important, it differentiated lesion components with high sensitivity and specificity.

The success of a supervised algorithm is directly determined by the selected training set. Thus, only clear-cut cases were used for this study while training the classifier, based on retrospective radiologic assessment for several months. Classification into tumor and nontumor components was performed based on conventional parameters (nT1WI+Gd, and nFLAIR) and parameters extracted from DCE $\left(v_{\mathrm{p}}, K^{\text {trans }}\right.$, and BAT). While perfusion parameters can be obtained using dynamic susceptibility contrast imaging and have been shown to differentiate between tumor and nontumor components, ${ }^{11-13,35,36}$ DCE imaging is preferable due to its higher spatial resolution, less sensitivity to susceptibility artifacts, and provision of quantitative parameters, including permeability $\left(K^{\text {trans }}\right)$.

Several issues are important when interpreting the clinical relevance of classification results. The relatively small sample size and absence of histology-proved diagnosis in our cohort call for additional studies. Tumor components were found to be highly vascular while the nontumor components were found to have reduced vascularity. However, the enhancing, nontumor component may represent a more complex situation. It may reflect treatment-related changes (such as radiation necrosis) but may also represent tumor-associated hy- 
poxic necrosis, which is one of the histologic hallmarks of GB. ${ }^{37}$ Thus, in such cases, it is important to consider the nontumor component in the context of active tumor surrounding the necrotic area.

\section{CONCLUSIONS}

The current study proposes a model of segmented RANO criteria, sRANO, that classifies tumor and nontumor components within a lesion area, with high sensitivity and specificity. Longitudinal assessment in patients demonstrated consistency between the classification results and radiologist's assessment in most cases; in $16 \%$ of patients, the segmentation results identified growth of highly vascular components and preceded the conventional radiologic diagnosis of tumor progression by several months. The proposed sRANO method and results presented in this study demonstrate the importance and contribution of segmentation of the enhancing and nonenhancing lesion areas into tumor and nontumor components, to improve therapy-response assessment of patients with malignant brain tumors.

\section{ACKNOWLEDGMENTS}

We are grateful to Vicki Myers for editorial assistance, to Faina Vitenstein and Tuvia Ganot for their assistance in patient recruitment and performing MRI scans, and to Dr Ricardo Tarrasch for statistical consulting.

Disclosures: Deborah T. Blumenthal—UNRELATED: Board Membership: Medical Advisory Board for Vascular Biogenics; Travel/Accommodations/Meeting Expenses Unrelated to Activities Listed: Roche, Bristol-Myers Squibb, Comments: travel grants to international meetings. ${ }^{*}$ Money paid to the institution.

\section{REFERENCES}

1. Stupp R, Mason WP, van den Bent MJ, et al; European Organisation for Research and Treatment of Cancer Brain Tumor and Radiotherapy Groups, National Cancer Institute of Canada Clinical Trials Group. Radiotherapy plus concomitant and adjuvant temozolomide for glioblastoma. $N$ Engl J Med 2005;352:987-96 CrossRef Medline

2. Friedman HS, Prados MD, Wen PY, et al. Bevacizumab alone and in combination with irinotecan in recurrent glioblastoma. J Neurooncol 2009;27:4733-40 CrossRef Medline

3. Wen PY, Macdonald DR, Reardon DA, et al. Updated response assessment criteria for high-grade gliomas: Response Assessment in Neuro-Oncology working group. J Clin Oncol 2010;28:1963-72 CrossRef Medline

4. Macdonald DR, Cascino TL, Schold SC Jr, et al. Response criteria for phase II studies of supratentorial malignant glioma. J Clin Oncol 1990;8:1277-80 Medline

5. Verma N, Cowperthwaite MC, Burnett MG, et al. Differentiating tumor recurrence from treatment necrosis: a review of neuro-oncologic imaging strategies. Neuro Oncol 2013;15:515-34 CrossRef Medline

6. Fink KR, Fink JR. Imaging of brain metastases. Surg Neurol Int 2013; 4(suppl 4):S209-19 CrossRef Medline

7. Fatterpekar GM, Galheigo D, Narayana A, et al. Treatment-related change versus tumor recurrence in high-grade gliomas: a diagnostic conundrum-use of dynamic susceptibility contrast-enhanced (DSC) perfusion MRI. AJR Am J Roentgenol 2012;198:19-26 CrossRef Medline

8. Sorensen AG, Batchelor TT, Zhang WT, et al. A "vascular normalization index" as potential mechanistic biomarker to predict survival after a single dose of cediranib in recurrent glioblastoma patients. Cancer Res 2009;69:5296-300 CrossRef Medline
9. Zhang W, Kreisl TN, Solomon J, et al. Acute effects of bevacizumab on glioblastoma vascularity assessed with DCE-MRI and relation to patient survival. In: Proceedings of the Scientific Meeting and Exhibition of The International Society for Magnetic Resonance in Medicine, Honolulu, Hawaii. April 18-24, 2009

10. Artzi M, Bokstein F, Blumenthal DT, et al. Differentiation between vasogenic-edema versus tumor-infiltrative area in patients with glioblastoma during bevacizumab therapy: a longitudinal MRI study. Eur J Radiol 2014;83:1250-56 CrossRef Medline

11. Barajas RF Jr, Chang JS, Segal MR, et al. Differentiation of recurrent glioblastoma multiforme from radiation necrosis after external beam radiation therapy with dynamic susceptibility-weighted contrast-enhanced perfusion MR imaging. Radiology 2009;253:486-96 CrossRef Medline

12. Barajas RF, Chang JS, Sneed PK, et al. Distinguishing recurrent intra-axial metastatic tumor from radiation necrosis following gamma knife radiosurgery using dynamic susceptibility-weighted contrast-enhanced perfusion MR imaging. AJNR Am J Neuroradiol 2009;30:367-72 Medline

13. Bobek-Billewicz B, Stasik-Pres G, Majchrzak H, et al. Differentiation between brain tumor recurrence and radiation injury using perfusion, diffusion-weighted imaging and MR spectroscopy. Folia Neuropathol 2010;48:81-92 Medline

14. Larsen VA, Simonsen HJ, Law I, et al. Evaluation of dynamic contrast-enhanced T1-weighted perfusion MRI in the differentiation of tumor recurrence from radiation necrosis. Neuroradiology 2013; 55:361-69 CrossRef Medline

15. Jensen TR, Schmainda KM. Computer-aided detection of brain tumor invasion using multiparametric MRI. J Magn Reson Imaging 2009;30:481-89 CrossRef Medline

16. Pope WB, Young JR, Ellingson BM. Advances in MRI assessment of gliomas and response to anti-VEGF therapy. Curr Neurol Neurosci Rep 2011;11:336-44 CrossRef Medline

17. Sourbron S, Ingrisch $M$, Siefert $A$, et al. Quantification of cerebral blood flow, cerebral blood volume, and blood-brain-barrier leakage with DCE-MRI. Magn Reson Med 2009;62:205-17 CrossRef Medline

18. Shah SA, Chauhan N. Techniques for detection and analysis of tumours from brain MRI images: a review. Journal of Biomedical Engineering and Medical Imaging 2016;3:9-20

19. Vanitha L, Venmathi A. Classification of medical images using support vector machine. In: Proceedings of the 2011 International Conference on Information and Network Technology, Nanjing, Jiangsu, China. September 24-25, 2011

20. Artzi M, Aizenstein O, Jonas-Kimchi $\mathrm{T}$, et al. FLAIR lesion segmentation: application in patients with brain tumors and acute ischemic stroke. Eur J Radiol 2013;82:1512-18 CrossRef Medline

21. Artzi M, Liberman G, Blumenthal DT, et al. Automatic Segmentation and Classification of Glioblastoma using DCE-MRI. In: Proceedings of the Annual Meeting and Exhibition of the International Society for Magnetic Resonance in Medicine, May 7-13, 2016. Singapore

22. Provencher SW. Estimation of metabolite concentrations from localized in vivo proton NMR spectra. Magn Reson Med 1993;30: 672-79 CrossRef Medline

23. Fogh SE, Andrews DW, Glass J, et al. Hypofractionated stereotactic radiation therapy: an effective therapy for recurrent high-grade gliomas. J Clin Oncol 2010;28:3048-53 CrossRef Medline

24. Dhermain F. Radiotherapy of high-grade gliomas: current standards and new concepts, innovations in imaging and radiotherapy, and new therapeutic approaches. Chin J Cancer 2014;33:16-24 CrossRef Medline

25. Pollack IF, Jakacki RI, Butterfield LH, et al. Immune responses and outcome after vaccination with glioma-associated antigen peptides and poly-ICLC in a pilot study for pediatric recurrent low-grade gliomas. Neuro Oncol 2016;18:1157-68 CrossRef Medline

26. Hardee ME, Zagzag D. Mechanisms of glioma-associated neovascularization. Am J Pathol 2012;181:1126-41 CrossRef Medline

27. Cloughesy TF, Cavenee WK, Mischel PS. Glioblastoma: from molec- 
ular pathology to targeted treatment. Annu Rev Pathol 2014;9:1-25 CrossRef

28. Artzi M, Liberman G, Nadav G, et al. Differentiation between treatment-related changes and progressive disease in patients with high grade brain tumors using support vector machine classification based on DCE MRI. J Neurooncol 2016;127:515-24 CrossRef Medline

29. Law M, Cha S, Knopp EA, et al. High-grade gliomas and solitary metastases: differentiation by using perfusion and proton spectroscopic MR imaging. Radiology 2002;222:715-21 CrossRef Medline

30. Uematsu H, Maeda M, Itoh H. Peritumoral brain edema in intracranial meningiomas evaluated by dynamic perfusion-weighted MR imaging: a preliminary study. Eur Radiol 2003;13:758-62 Medline

31. Hossman KA, Blöink M. Blood flow and regulation of blood flow in experimental peritumoral edema. Stroke 1981;12:211-17 CrossRef Medline

32. Kaal EC, Vecht CJ. The management of brain edema in brain tumors. Curr Opin Oncol 2004;16:593-600 CrossRef Medline

33. Sentürk S, Oğuz KK, Cila A. Dynamic contrast-enhanced suscepti- bility-weighted perfusion imaging of intracranial tumors: a study using a 3T MR scanner. Diagn Interv Radiol 2009;15:3-12 Medline

34. Al-Tamimi MSH, Sulong G. Tumor brain detection through MR images: a review of literature. Journal of Theoretical and Applied Information Technology 2014;62:387-403

35. Hu LS, Baxter LC, Smith KA, et al. Relative cerebral blood volume values to differentiate high-grade glioma recurrence from posttreatment radiation effect: direct correlation between image-guided tissue histopathology and localized dynamic susceptibility-weighted contrast-enhanced perfusion MR imaging measurements. AJNR Am J Neuroradiol 2009;30:552-58 CrossRef Medline

36. Kim J, Leira EC, Callison RC, et al. Toward fully automated processing of dynamic susceptibility contrast perfusion MRI for acute ischemic cerebral stroke. Comput Methods Programs Biomed 2010;98: 204-13 CrossRef Medline

37. Noch E, Khalili K. Molecular mechanisms of necrosis in glioblastoma: the role of glutamate excitotoxicity. Cancer Biol Ther 2009;8:1791-97 CrossRef Medline 